\title{
Magia y tradición: Un ejemplo homérico ${ }^{1}$
}

1. En algunos relatos mitológicos brujas y magas han empleado hierbas en sus hechizos y brebajes. La dificultad que plantea la identidad y uso de estas plantas ha sido objeto de estudio de numerosos especialistas, que han propuesto diversas hipótesis al respecto. En el marco de esta problemática se incluye la siguiente contribución, que trata de dar una nueva visión en torno a dos palabras cuyo análisis ha sido controvertido.

El canto X (vs. 285ss) de la Odisea nos narra lo acaecido a los compañeros de Odiseo en la isla Eea, donde son hechizados con un brebaje de hierbas preparado por la maga Circe, que los transforma en cerdos. Uno de ellos, Euríloco, logra escapar a la pócima maléfica de Circe advirtiendo de este peligro a Odiseo. Éste, que se pone en camino hacia la mansión donde están presos sus amigos con el objetivo de liberarlos, se encuentra con el dios Hermes quien proporciona a Odiseo una planta mágica que le inmuniza de los hechizos de Circe.

En este breve episodio, hemos de dirigir nuestra atención, por un lado

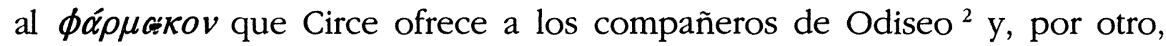
a la planta que Hermes da a Odiseo, llamada en el texto original $\mu \hat{\omega} \lambda v$ y descrita como: "de raíz negra y flor parecida a la leche, difícil de arrancar para los hombres mortales, más no para los dioses, que todo lo pueden" ${ }^{3}$.

Sobre el $\phi \alpha ́ \rho \mu \alpha \kappa o v$ creemos que pueden existir diversas hipótesis:

a) que, al ser la hierba utilizada por Circe (hierba de Circe), se la relacione con el género Circea $^{4}$, nombre tal vez derivado del de la diosa, sólo descrita en Dioscórides en De Materia Medica CXXVIII, 349, o;

b) que sea la Mandrágora ${ }^{5}$, que, como dice Dioscórides: “... algunos la llaman Antimalo y otros Circea porque su raíz parece útil a los hechi-

1 Este trabajo, realizado en el marco del Proyecto de Investigación DGICYT 93-0254.

2 Cf. Od. X 287.

Cf. Od. X 304-5.

4 Cf. también Reinhold STRÖMBERG, Griechische Pflanzennamen (Göteborg, 1940), p. 93.

5 La etimología de $\mu \alpha v \delta \rho \alpha \gamma o ́ \rho \alpha \varsigma$ es incierta. Pierre Chantraine, Dictionnaire étymologique de la langue grecque: Histoire des Mots (Paris, 1983-84), p. 664 y Hjalmar 
zos". Plinio, Naturalis Historia, XXV XCIV.147 avala esta opinión, afirmando que la Mandrágora se llama también Circea.

Por su parte, $\mu \hat{\omega} \lambda v^{6}$ ha sido objeto de una mayor atención por los diferentes autores: así, Teofrasto Historia de las plantas, IX XV.7 y Dioscórides De Materia Medica, III 299 describen un género Moly, que identifican con la planta homérica. Autores posteriores han tratado de compararla con la Ruda salvaje. Plinio, Naturalis Historia, XXV VIII.26 dice además, que los escritores griegos pensaban que era una especie de Allium amarillento.

2. A la luz de los datos que nos ofrecen los diferentes autores clásicos, la investigación sobre la identidad de estas plantas parece haber constituido un problema ciertamente complejo, lo que nos lleva a plantear una serie de cuestiones:

- Partiendo de las descripciones dadas por los antiguos para Circea y la Mandrágora, ¿cabe concluir que sean la misma planta?; ¿Se puede

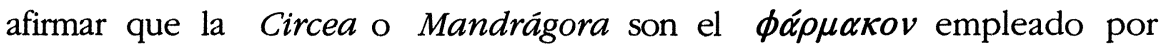
Circe?

- Tomando como referencia las características que de $\mu \hat{\omega} \lambda v$ hay en los textos, ¿sería posible encontrar la verdadera identidad de esta planta,

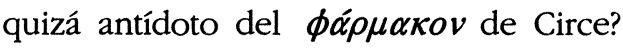

3. Con respecto a la primera pregunta, contrastemos cómo describe Dioscórides la morfología de estas dos plantas:

La Circea es una planta con hojas parecidas a las de la Hierba Mora ${ }^{7}$, mientras que las de la Mandrágora, hembra y macho ${ }^{8}$, son semejantes a las de la acelga o lechuga y, además, las de la hembra desprenden "un grave olor".

La Circea tiene flores negras, numerosas y pequeñas, y de la flor de la Mandrágora no hay descripción por parte de Dioscórides, aunque Pío Font Quer en su Dioscórides Renovado nos dice que tanto la hembra (Mandragora autumnalis) como la macho (Mandragora vernalis u officina-

FRISK, Griechische Etymologisches Wörterbuch (Heidelberg, 1954-72), p. 170 afirman que el término "mandrágora" podría ser un préstamo del a.persa. merdum gija 'planta humana'.

6 En Chantraine y Frisk se pone en relación la planta con scr. múlam 'raíz' (RV+), de oscura etimología, cf. Manfred MAYRHOFER, Kurzgefasstes etymologisches Wörterbuch des Altindischen (Heidelberg, 1956-1970-1979), p. 667.

7 Cuyas hojas son ovales y estrechas.

8 En la Mandrágora se distinguen dos especies diferentes: la hembra de raíz blanca y la macho de raíz negra. 
rum) tienen flores que nacen en el centro de un rosetón, con cinco pétalos azul-violáceo.

Por lo que respecta al fruto, la Circea lo tiene en forma de vaina (como un cornezuelo), mientras que el de la Mandrágora es como una pequeña manzana amarillenta.

Finalmente, Dioscórides nos describe la raíz de la Circea, de color blanco y ramificada como la de la hembra de la Mandrágora, y no como la del macho, que la tiene negra 9 .

El contraste de las descripciones de la Circea y la Mandrágora dadas por los diferentes autores, nos demuestra que morfológicamente son distintas. Pero veamos los usos descritos para estas plantas:

Sobre la Circea, Dioscórides comenta algunos de sus usos medicinales: que su raíz tiene fuerza de calentar y metida en vino dulce purga la madre. Y que su simiente, disuelta en potajes, acrecienta la leche. Además, ciertos relatos mitológicos recogen usos mágicos de esta planta que la relacionan de nuevo con Circe, así Apolodoro, Biblioteca, III 15.1 nos narra el mito de Pasífae, Minos y Procris donde Circe proporciona a Procris, amante de Minos, la raíz Circea para liberarle del hechizo de su esposa Pasífae.

Por lo que respecta a la Mandrágora, Teofrasto, Historia de las plantas, IX 8.8, nos habla de sus propiedades afrodisíacas ${ }^{10}$. Por su parte, Dioscórides dice que las raíces cocidas en vino ayudan a los que no pueden dormir ${ }^{11}$ o tienen algún dolor, opinión apoyada por Hipócrates, Los lugares del hombre, XXXIX 1, quien prescribe, a la gente afligida enferma y débil, el uso de la raíz, bebida por la mañana en dosis pequeñas para no producir locura. Siguiendo con Dioscórides, éste enumera otras propiedades de la planta: su raíz atrae el menstruo y el parto, es buena

9 PLINIO, Historia Natural XXV 94 añade a la descripción de la Mandrágora, que tiene las raíces trenzadas y largas de más de un codo de largo. La variedad macho tiene hojas verde-blanquecino parecidas a las de la hierba de la paciencia y más largas que las de la hembra; los frutos de ambas son del tamaño de avellanas y su simiente es como un pepino envainado.

10 En la Biblia se menciona en dos ocasiones esta propiedad de la Mandrágora: Génesis XXX 14-18 en donde Raquel pide a su hermana Lía las mandrágoras que ha arrancado su hijo, permitiendo, a cambio, que Lía durmiera con Jacob esa noche, en la que concibió su quinto hijo. Cantar de los Cantares VII 13 "... Ya despiden su fragancia/ las mandrágoras;/ junto a nuestras puertas/ hay toda clase de frutas exquisitas;/ las nuevas y las pasadas/ he guardado, amado mío, para tì.

11 JENOFONTE, Banquete XXIV-XXV recoge el diálogo que mantiene Sócrates con sus amigos, en el que se describe el poder soporífero de la Mandrágora: «Tambien a mí me parece muy bien el beber, amigos, pues en realidad el vino al regar las almas adormece las penas, como la mandrágora hace con los hombres. 
medicina para ojos ${ }^{12}$; pero, si se bebe en gran cantidad, es mortífera. Plinio, Historia Natural, XXV 94 añade que la violencia del perfume de la mandrágora aturde a los que no están hechos para olerla (cf. infra, el ritual de las lemnias), que el jugo de las plantas frescas es un peligroso veneno y afecta a la cabeza.

Como en el caso de la Circea, exiten otras historias que hacen referencia al empleo de la Mandrágora: Mírsilo de Metimna, en su obra sobre Lesbos, nos narra que la hechicera Medea (sobrina de Circe y Pasífae) al pasar junto a Lesbos en compañía de Jasón, había arrojado en ella $\phi \alpha ́ \rho \mu \alpha \kappa \alpha$ que fueron la causa del repugnante olor de las mujeres de la isla. Este mito se corresponde con el Ritual de la fiesta lemnia ${ }^{13}$, donde las mujeres cada año eran separadas de los hombres a causa del mal olor que ellas emanaban. La interpretación de este rito era que Afrodita, llamada por una glosa 'mandragoritis', las había castigado con este nauseabundo olor al sentirse despreciada por ellas, condenándolas a la ruptura de sus relaciones conyugales y a la putrefación.

Como se ha comprobado, tanto en la Circea como en la Mandrágora, la raíz es empleada para realizar curaciones o hechizos.

Para poder responder a la pregunta de si Circea o Mandrágora son la misma planta partimos de la base de que tenemos dos plantas morfológicamente distintas, que sólo tienen un punto en común: la utilización médica y mágica de su raíz.

Proponemos, como hipótesis, que la planta Circea y los usos de su raíz fueran conocidos en Grecia con anterioridad a los de la Mandrágora, puesto que ésta proviene de Oriente ${ }^{14}$. Luego, es posible que se empleara el nombre Circea a todas aquellas raíces de plantas utilizadas en brujería y hechizos y por extensión, al resto de la planta. Así se explica lo que Dioscórides nos dice de la Mandrágora que también es llamada Circea "porque su raíz parece útil a los filtros mágicos" (Dioscórides, IV 75 y III 233).

Como apoyo a nuestra teoría encontramos el siguiente paralelismo en Schrader-Nehring ${ }^{15}$, donde se dice que antiguamente, en Alemania, existía una palabra, Alruna: "que conoce todos los misterios", que, según creencia pagana, hacía mención al ser mágico femenino: Idise y otras. Poste-

${ }_{12}$ Se dice que la raíz con aceite de rosa y vino cura el dolor de los ojos, cf. PuINIO, Historia Natural, loc. cit..

13 Cf. Marcel DetIENNE, Les jardin d'Adonis. La mythológie des aromates en Gréce (Paris, 1982).

14 Cf. el origen propuesto por los etimológicos al uso.

15 SCHRADER-NEHRING, Reallexicon der Indogermanischen Alterstumskunde (Berlin, 1917), pp. 42-3. 
riormente, cuando se introdujo en Alemania la mandrágora y el culto que la envolvía, el significado de Alruna también hizo referencia a la magia de ciertos idolillos masculinos y femeninos creados a partir de la raíz de una planta que en glosas del antiguo alto alemán se identificó con lat. mandragora ${ }^{16}$.

En el Diccionario "Der grosse Herder": se dice que Alraun, derivado del Alruna de Schraeder, es una raíz o rizoma de determinadas plantas, que tiene figura humana y a la que la superstición popular atribuye virtudes mágicas, como las del Allium victorialis, de la Bryonia cretica y sobre todo de la Mandragora officinarum de los países mediterráneos. Por esta razón en Alemania a la Mandrágora se la llama Alraun ${ }^{17}$.

En conclusión, creemos que el género Mandrágora y Circea son diferentes, pero la Mandrágora quedó englobada por sus usos mágicos dentro de la denominación general Circea. Según esta explicación podríamos

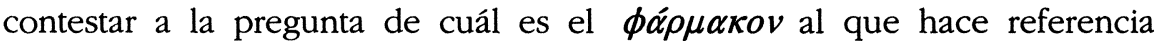
Homero.

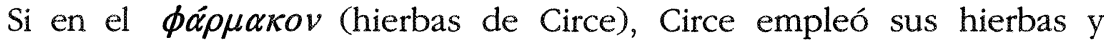
raíces y el término Circea engloba, como hemos visto, a todo tipo de raíces que tienen poderes mágicos, cabe como posibilidad proponer que la Mandrágora o raíz Circea, fuera el componente o uno de los ingredientes utilizados en el $\phi \alpha ́ \rho \mu \alpha \kappa o v$ que convirtió en cerdos a los amigos de Odiseo ${ }^{18}$.

4. Por lo que respecta a la segunda cuestión acerca de $\mu \hat{\omega} \lambda \nu$, hagamos un recorrido a lo largo de diferentes autores en busca de la verdadera identidad de esta planta.

Teofrasto, loc. cit., nos dice que $\mu \hat{\omega} \lambda v$ es una planta que nace en lugares rocosos en Feneo y en Arcadia, en el monte Cilene, y que se utiliza contra la brujería y los hechizos, y afirma que es como la planta mencionada por Homero, con raíz redonda, como un ajo y una hoja como la de la Scilla maritima. Pero no es difícil de arrancar en contraposición a la descripción homérica.

Dioscórides, loc. cit., amplía esta descripción y comenta que tiene las hojas más anchas que la grama y derramadas sobre la tierra. Que sus flo-

16 La forma del lituano kaukas, significó 'hada' inicialmenmte y derivó después en 'mandrágora', cuando el culto a esta planta fue introducido en los paises baltoeslavos.

17 Véase la siguiente proporción: Alrune $=$ ser mágico femenino $=$ idolillos $=$ raíz mágica $=$ Alraun $=$ Mandrágora .

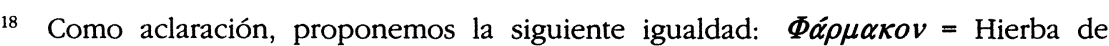
Circe $=$ raíz Circea $($ por derivación del nombre de la diosa $)=$ Mandrágora . 
res son del color de la leche, semejantes a las del alhelí, con tallo alto de cuatro codos y ápice parecido al del ajo. Además, en una anotación confirma que es la planta homérica, aunque algunos la confunden con la Ruda silvestre.

Plinio, Historia Natural, XXV 7 recoge lo de Teofrasto y dice que su descubrimiento y propiedades contra venenos maléficos son atribuidos a Hermes ${ }^{19}$. Así mismo, afirma que los escritores griegos representaban una especie de ajo de flor amarilla que se creía brotaba cuando menguaba la luna, de ahí que sirviera como contraencantamiento de la magia lunar de Hécate ${ }^{20}$, por eso, Murr interpreta que era una especie de Allium amarillento.

4.1. La comparación que establecen Teofrasto y escritores griegos (cf. supra Plinio) de $\mu \hat{\omega} \lambda v$ con una especie de ajo, ha llevado a distintos autores a identificar $\mu \hat{\omega} \lambda v$ con el género Allium.

Dioscórides describe en su tratado las siguientes especies de Allium: el puerro cabezudo ${ }^{21}$ (CXXXVIII 229), puerro salvaje ${ }^{22}$ (CXXXIX 230,), la cebolla ${ }^{23}$, ajo doméstico (CXII 231), que es blanco como el puerro ${ }^{24}$, opbioscorodon o ajo salvaje (CXLI 234), con flor bermeja y, por último, el scorodoprassum $^{25}$ (CXIII 233), a las que atribuye diversas propiedades: alucinógenas, afrodisíacas, contra venenos etc.

En cambio, Murr, en su estudio, no identifica ninguna de estas especies con $\mu \hat{\omega} \lambda \nu$, sino que estudia otras como el Allium victorialis (puerro de los bosques alemanes, llamado "armadura de Allermann", al servir de protección contra las heridas) y el Allium moly, que también desestima por no encontrarse ninguna de ellas en Grecia ${ }^{26}$. Murr estaba convencido de que $\mu \hat{\omega} \lambda v$ era una especie de puerro, porque a éstos se les atribuía,

19 Hermes fue alumbrado por la ninfa Maya en una cueva del monte Cilene.

20 Según Josef MURR, Die Pflazenwelt in der Griechischen Mythologie (Groningen, 1969), p. 202, la mandrágora aparece plantada en el jardín mágico de Hécate en la Cólquide; Circe puede ser hija de Hécate y hermana del rey de la Cólquide, Eetes, y de Pasífae (esposa de Minos), cf. FALCón MARTínez, et. al., Diccionario de Mitología Clásica I (Madrid, 1988), p. 146.

21 Allium porrum.

22 Allium ampeloprason, con flores blanquecinas y purpúreas, cf. PoLUNIN 1982: 577.

23 Allium cepo, flores blanco-verdosas, cf. O. Polunin, Guia de campo de las flores de Europa (Barcelona, 1982), p. 574.

24 Allium sativum, flores blanquecinas, cf. POLUNIN, op. cit., p. 576.

25 Allium scorodoprasum, flores rojo purpúreas, cf. POLUNIN, op. cit., p. 576.

26 Cf. MURR, op. cit., p. 203. 
a causa de su penetrante sabor y olor, la capacidad de romper terribles encantamientos e inutilizar los venenos, e identifica $\mu \hat{\omega} \lambda \nu$ con el Allium neapolitanum $^{27}$, que crece en Grecia y en las islas, o con el Allium subbirsutum ${ }^{28}$, que también está en toda Grecia.

A la vista de lo expuesto, la identificación de $\mu \hat{\omega} \lambda \nu$ con una especie de Allium no ha llevado a una conclusión definitiva, sino a múltiples posibilidades.

4.2. La otra posibilidad planteada para $\mu \hat{\omega} \lambda \nu$ es la ruda:

Dioscórides, De materia medica, II 48 describe los usos de varias especies: la $R$. montana y $R$. doméstica ( $R$. graveolens) ${ }^{29}$ : de las que dice que son antiespermáticas en el varón. La raíz es llamada moly montano y metida en un higo con media nuez y sal es antídoto contra todo veneno y fuerza contra toda suerte de hechicerías y malos espíritus. Para arrancar b $R$. montana ${ }^{30}$ en flor, se recomienda ponerse aceite en las manos y cara, porque produce irritación. De la $R$. salvaje, describe que tiene las hojas más largas que las de la $R$. montana, con fuerte olor. Se llama Harmala y Besasa en Siria, y en Capadocia, Moly por parecerse algo a $\mu \hat{\omega} \lambda v^{31}$, al tener como ésta la raíz negra y blanca la flor.

En consonancia con los usos descritos por Dioscórides, las creencias en torno a las propiedades mágicas (contra la brujería y los maleficios) de la ruda se han mantenido a lo largo de la historia en numerosas regiones del mundo: así, ya los romanos comían la ruda en ensalada para inmunizarse contra los venenos. En Europa Central se le atribuye la propiedad de ahuyentar a las brujas. En Marruecos, se utiliza como curativo y contraveneno y en Argel se emplea para anular el apetito sexual. Por último, en el Pirineo aragonés, la gente, con el dedo impregnado en aceite, hace la señal de la cruz en una parte del cuerpo, a la vez que recita "con aceite y ruda no entrarás mala bruja".

27 Con flores numerosas completamente blancas, cf. Polunin, op. cit., p. 576.

28 Con flores blancas en umbela floja y patente, cf. Polunin, op. cit., p. 575.

29 En Polunin, op. cit., p. 262 se describe así la $R$. graveolens: es aromática con hojas compuesta verde-amarillentas, con glándulas aromáticas. Tiene ramilletes de flores amarillas y crece en zonas rocosas y secas.

30 Cf. P. RIBERA NÚÑEZ y C. OBÓN DE CASTRO, Guía de INCAFO de las plantas útiles $y$ venenosas de la Península Ibérica y Baleares (Barcelona, 1992), pp. 695 y ss: glándulas hacia la punta, hojas divididas en hojillas, flores pequeñas y amarillas, frutos como cápsulas y en racimos, crece en zonas pedregosas y áridas.

31 MURR, op. cit., p. 210 afirma que en el norte de Asia hay dos plantas con el nombre de $R$. graveolens y $R$. montana que reciben el nombre de $\mu \hat{\omega} \lambda v$. 
5. Tomando como referencia la descripción homérica y ateniéndonos siempre a las plantas propuestas por estos autores, discutamos a continuación la identidad de $\mu \hat{\omega} \lambda \nu$, entendiéndola como un antídoto contra el $\phi \alpha ́ \rho \mu \alpha \kappa o v$ de Circe, la Mandrágora. Por consiguiente, hemos de buscar

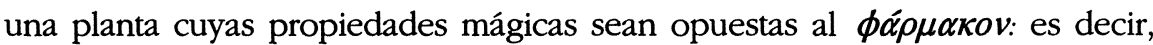
contra maleficios y brujería, antiafrodisíaca, y, además, que su forma concuerde con la descrita por Homero.

Entre las plantas que tenemos como opción y que se ajustan a las condiciones propuestas, se encuentran el Allium subbirsutum, el Allium neapolitanum y por último, la Ruta (quizás la $R$. salvaje). Para poder determinar cuál de las tres plantas se identifica con $\mu \hat{\omega} \lambda \nu$, hemos de encontrar otros argumentos que permitan buscar una solución adecuada: En nuestra opinión, cuando los antiguos comparaban $\mu \hat{\omega} \lambda \nu$ con una especie de ajo, lo hacían para su mejor identificación, lo que no quiere decir que $\mu \hat{\omega} \lambda v$ fuera un Allium, sino que se parecía a él ${ }^{32}$. Además, lo que llama la atención en Dioscórides es que, mientras el Allium no recibe la denominación de $\mu \hat{\omega} \lambda \nu$, sí la tiene la $R$. salvaje ${ }^{33}$, no sólo por sus usos, sino por su apariencia física, lo cual coincide con el mismo esquema que en el caso del $\phi a ́ p \mu \alpha \kappa o v$, en el que Mandrágora recibía el nombre de Circea por los usos mágicos de su raíz.

En consecuencia, es lícito resolver que la planta $\mu \hat{\omega} \lambda \nu$, que Hermes dio a Odiseo, fue en realidad la llamada $R$. salvaje. Como no se ha encontrado en los libros consultados una planta del género Ruta que se adecúe a la descripción dada por Dioscórides para $R$. salvaje, ésta se ha tratado de identificar con la Peganum harmala, que se usa en el norte de África para descubrir los males de ojo y se cuelga en las casas como protección contra los malos genios. Además, en Marruecos dicha planta, que tiene propiedades mágicas similares a las de la ruda, es utilizada como contraveneno. Pensamos que es posible que esta relación sea debida a que $\pi \dot{\eta} \gamma \alpha v o v$ en griego es 'ruda', y Harmala es el nombre de la $R$. salvaje en Siria.

6. Las conclusiones más importantes que se desprenden de este artículo quedan resumidas como sigue:

(1) La Mandrágora, que también recibe el nombre de Circea, fue el componente o uno de los ingredientes del фа́́ $\mu \alpha \kappa o v$ empleado por la maga Circe para hechizar a los compañeros de Odiseo.

32 Baste recordar que Teofrasto compara la hoja de $\mu \hat{\omega} \lambda \nu$ con la de la Scilla maritima y no por ello la identifica con ésta.

33 Recordemos que a la $R$. montana también se le llamó moly montano por su raíz negra, pero ésta no es la descrita por Homero, ya que sus flores son amarillas. 
(2) La $R$. salvaje puede ser identificada con la planta $\mu \hat{\omega} \lambda v$ descrita por Homero y utilizada como contraencantamiento del $\phi \alpha ́ \alpha \mu \alpha \kappa o v$, por su forma, propiedades y denominación.

\author{
José Manuel Cuesta Pastor \\ YVONNE PASTOR SECO
}

El mundo de la brujería y la magia aparece en ocasiones en la mitología; en este caso, en la Odisea, la bruja Circe envenena a los amigos de Odiseo convirtiéndolos en cerdos, y Hermes proporciona a éste el antídoto para poder rescatarlos. Con el apoyo de consultores botánicos antiguos y la tradición popular y mitológica se tratará de demostrar la identidad del phármakon de la maga que esconde en realidad una planta misteriosa: la mandrágora. El mismo procedimiento se seguirá para la identificación de moly, una planta que nace en el monte Parnaso con propiedades curativas semejantes a las de la ruda.

The world of witchcraft and magic sometimes appears in mythology; for example in Odyssey, the witch Circe poisons Odysseu's friends, transforming them into pigs and Hermes provides the antidote which saves them. With the support of the old botanical consultants and the popular tradition and mythology we will try to demonstrate the identity of pharmakon of the witch, which in fact conceals a mysterious plant. The same proceedure will be followed in the identification of moly - a plant found in the Parnaso mountain with healing properties similar to those of the ruda. 